\title{
Generisch, unpersönlich, indefinit? Die Pronomina man, on, one und generisches you im politischen Diskurs
}

„Wer ist denn man?“ (Mais qui est on ?) - so beginnt Viollets Beitrag (1988: 67) über den Referenzbezug des französischen Pronomens on. Trotz sprachspezifischer Merkmale haben seine „Äquivalente“ im Deutschen (man) sowie im Englischen (one) eine ähnliche Sonderstellung (Zifonun 2000: 232), die vielleicht auch erklären kann, warum diese Pronomina aus pragmatisch-diskursiver Perspektive immer noch ungenügend untersucht worden sind. Gerade der sonderbare referenzielle Bezug dieser Pronomina, die mit einer ganzen Reihe von Eigenschaften versehen werden können (generisch, unspezifisch, indefinit, unpersönlich, u.a.), macht sie für politische Sprecher/innen zu interessanten Markierern, da durch ihre Verwendung häufig nicht klar wird, wer tatsächlich gemeint ist.

Genau diese referenzielle Instabilität ist Thema des vorliegenden Beitrags. Dabei wird der empirische Fokus auf Parlamentsdebatten in Deutschland, Großbritannien und Frankreich gelegt. Indem der Gebrauch der Pronomina man, on, one und generisches you systematisch verglichen wird, werden sowohl gemeinsame Funktionen der Pronomina über Nationalgrenzen hinaus, als auch Unterschiede, die mit dem Sprachsystem oder mit der politischen Kultur zusammenhängen, vor Augen geführt.

Aus einer sprachvergleichenden Perspektive ist es in erster Linie wichtig, morpho-syntaktische und semantische Aspekte der Pronomina man, on, one und generisches you kurz darzustellen. Darauffolgend wird auf Referenzkonstruktionen in Parlamentsdebatten eingegangen: Das Genre parlamentarischer Debatten bestimmt die potenziellen Referenten der Pronomina. Zunächst werden quantitativ gewonnene empirische Ergebnisse der Korpusuntersuchung präsentiert. Anschließend werden die Daten mit einem qualitativen Blick betrachtet. Eine Typologie der möglichen referenziellen Funktionen der Pronomina man, on und one und generisches you im politischen Diskurs stellt die Grundlage für die qualitative Untersuchung von drei Hauptwerten: der allgemeine Personenbezug, der selbstreferenzielle Erfahrungsbezug und der Bezug auf andere. Rückblickend werden die in der Forschung erwähnten Aspekte von man, on und one und generisches you anhand des spezialisierten Korpus von Parlamentsdebatten neu bewertet: Inwiefern sind man, on und one generisch, unpersönlich, indefinit? 


\section{Die Pronomina man, on, one und generisches you im Vergleich: Morpho-syntaktische und semantische Aspekte}

Pronomina der 3. Person wurden aus einer monolinguistischen (siehe u.a. Wales 1980; Mignot 2015 für one; Zifonun 2000 für man; Viollet 1988; Fløttum/Jonasson/ Norén 2007; Mayaffre 2013 für on) sowie aus einer sprachvergleichenden (Bertrand 2002; Altenberg 2005; Malamud 2012) oder typologischen Perspektive (Haspelmath 2001) erforscht. In der Forschung wurden auch die generischen Funktionen des englischen Pronomen you (Kitagawa/Lehrer 1990; Gast et al. 2015) untersucht.

Genau wie on (Viollet 1988: 68) bleibt man auf die Subjektfunktion beschränkt (Zifonun 2000: 232). ${ }^{1}$ One kann zwar drei Wortklassen entsprechen (Personalpronomen, Nominalphrase, Determinativ, siehe Payne/Huddleston, 2002: 386); jedoch wird im vorliegenden Beitrag one ausschließlich als Personalpronomen betrachtet. In diesem Fall kommt one auch exklusiv als Subjekt vor. Aufgrund des beschränkten Gebrauchs des Pronomens one, das überwiegend in formellen Zusammenhängen auftaucht (Quirk et al. 1985: 353), wurde das Pronomen you ebenfalls in seiner generischen Lesart berücksichtigt, weshalb im britischen Korpus beide Formen miteinander verglichen werden können. Generische Interpretationen von Pronomina der 2. Person im Deutschen und im Französischen sind gleichfalls belegt (siehe u.a. Kluge 2016). Da jedoch die Pronomina auch besonders häufig in einer deiktischen Lesart vorkommen, wurden sie aus praktischen Zwecken aus dieser Studie ausgeschlossen. Im britischen Korpus ist die Zahl des Pronomens you (sowohl generisch als auch deiktisch) sehr niedrig, da die Anredeform durch die 2. Person im House of Commons verboten ist (Ilie 2005: 179).

Wie Johansson (2007: 185) ausführt, ist man stilistisch neutraler und entspricht gleichzeitig dem formellen one und dem informellen you im Englischen. On und man sind etymologisch verbunden: Das französische on lässt sich vom Lateinischen homo herleiten, das deutsche man vom Substantiv Mann; one weist wiederum auf das Numerus hin (Old English an, siehe Rissanen 1967: 5). Semantisch können alle drei Pronomina nur auf Menschen Bezug nehmen. Im Gegensatz zu man und one, die beide generisch ${ }^{2}$ verwendet werden können, kann das

1 Aufgrund einer teilweise fehlerhaften Tokenisation wurden die Akkusativ- und Dativformen von man, nämlich einen und einem, aus dieser Studie ausgeschlossen. Diese Entscheidung dient auch der Vergleichbarkeit der Daten.

2 Eine „partikuläre Verwendung“ von man im Sinne von irgendjemand, irgendwelche Leute ist auch möglich (Zifonun 2000: 237); sie ist aber im Korpus nicht belegt. Diese Ergebnisse 
Pronomen on als informelles wir benutzt werden. Da dieser Gebrauch im Korpus jedoch nicht belegt ist - was die Besonderheit der Parlamentsdebatten im Vergleich zu anderen Genres unterstreicht - wird auf diese Verwendung nicht weiter eingegangen. Nachdem spezifische Eigenschaften der Pronomina in den jeweiligen Sprachen aufgelistet wurden, werden man, on und one und generisches you im politischen Diskurs untersucht.

\section{Referenzkonstruktion in Parlamentsdebatten: Über die Vielzahl der möglichen Referenten}

Der breite semantische Umfang der Pronomina geht mit einer Vielzahl möglicher Referenten einher. Jedoch bedeutet diese Unbestimmtheit nicht, dass die Interpretation nicht kontextuell begrenzt werden kann - eigentlich ist genau das Gegenteil der Fall. Im politischen Diskurs insbesondere zielen die Pronomina man, on und one und generisches you häufig auf spezifische, leicht erkennbare Referenten. Dieser Beitrag zeigt, inwieweit im Parlament zwei verschiedene Kategorien von Rezipient/innen anhand der Pronomina man, on und one verwendet und in die Sichtweise des/der Abgeordneten integriert werden.

In Anlehnung an Ducrot (1980: 43f.) scheint der Unterschied zwischen Adressaten (allocutaires) und Adressierten (destinataires) von großer Bedeutung zu sein. Während Adressaten prototypisch durch die 2. Person enkodiert werden, bezeichnet der Begriff Adressierte jene Personen, die im Hinblick auf den Sprechakt und dessen illokutive Kraft adressiert werden. Wenn die Definition von Adressierten auch linguistische Markierer - und nicht nur den Verweis auf die illokutive Kraft - einbezieht, dann kann gerade die 3. Person auf Adressierte hinweisen. Anders gesagt können die Referenten der Pronomina man, on, und one und generisches you zwar nicht formell als Adressaten angesehen werden; jedoch verweisen sie auf eben jene Personen, die zu den Adressierten des politischen Diskurses gehören. Mit Adressierten können zwei Hauptgruppen gemeint sein: entweder das anwesende Publikum, das meistens aus Abgeordneten besteht, ${ }^{3}$ oder das abwesende Publikum, das Bürger/innen als Repräsentation einer wahrgenommenen Gruppe und nicht als konkrete realweltliche Personen umfasst.

stimmen mit Zifonuns Erkenntnissen (ebd.: 240) überein: „generisches man [überwiegt] eindeutig“.

3 Im Publikum können auch Journalist/innen sowie angemeldete Bürger/innen anwesend sein. Sie spielen aber eine geringe Rolle für Parlamentsdebatten im Allgemeinen und für den Referenzbezug der Pronomina man, on und one und generisches you eine noch geringere Rolle. 
Dabei wird gezeigt, dass der unbestimmte Referenzbezug der Pronomina sowohl auf andere Abgeordnete als auch auf eine weiter reichende Öffentlichkeit zielen kann. Diese Spannung ist konstitutiv für Parlamentsdebatten, weil selbige formell an die Abgeordneten gerichtet sind - die linguistisch gesehen die Adressatengruppe bilden und durch prototypische Markierer der 2. Person angesprochen werden ${ }^{4}$ - und dabei gleichzeitig Bürger/innen in Anspruch nehmen, die aber aus einer linguistischen Perspektive als Adressierte gelten und durch das Paradigma der 3. Person einbezogen werden.

Trotz intensiver Forschung über die Pronomina der 1. Person Plural (wir) sowie der 2. Person ( $d u$, ihr) im politischen Gebrauch sind weitere linguistische Untersuchungen über die Pronomina der 3. Person notwendig. Parlamentsdebatten rücken in den letzten Jahren immer wieder in einer sprachvergleichenden Perspektive (Bayley (Hg.) 2004; Ilie (Hg.) 2010) in den Fokus von Sprachwissenschaftlern/innen (Burkhardt/Pape 2000); sie bleiben aber noch weitgehend unerforscht (Gelabert-Desnoyer 2008: 410), insbesondere in einer qualitativ-quantitativen Perspektive.

Durch die vorliegende Korpusanalyse soll ein Beitrag dazu geleistet werden. Das Korpus ${ }^{5}$ besteht aus nationalen Plenardebatten zwischen 1998 und 2015, die kurz vor oder nach einer Zusammenkunft des Europäischen Rates abgehalten wurden. Reguläre Sitzungen des Europäischen Rats finden viermal im Jahr statt. Pro Jahr wurde eine Sitzung des Europäischen Rates nach qualitativen Kriterien ausgewählt, so dass das Korpus eine Reihe von wichtigen, konfliktreichen Parlamentsdebatten über europäische Themen beinhaltet. Die doppelte Einschränkung auf Diskursgenre (Parlamentsdebatten) und Thema (Europa) dient der Vergleichbarkeit der deutschen, französischen und britischen Subkorpora. Im Vergleich zu großen Referenzkorpora wird das Korpus folgender Arbeit als „kleines spezialisiertes Korpus“ beschrieben (Koester 2010).

Das Korpus wurde hinsichtlich folgender Metadaten gemäß der Text Encoding Initiative (TEI) Guidelines ${ }^{6}$ manuell annotiert: Sprecher/in, Geschlecht,

\footnotetext{
$4 \mathrm{Im}$ britischen House of Commons werden aber Abgeordnete durch die 3. Person angesprochen (siehe Ilie 2005: 179 in Abschn. 1).

5 Das Korpus besteht aus drei Subkorpora. Sie sind mit einer vollständigen Dokumentation (auf Englisch) online abzurufen und werden hier aus Platzgründen nur grob dargestellt: https://hdl. handle.net/11403/de-parl (Deutschland), https://hdl.handle.net/11403/fr-parl (Frankreich), https://hdl.handle.net/11403/uk-parl (Großbritannien) (Stand: 1.7.2017).

6 Die Text Encoding Initiative (TEI) ist ein Dokumentenformat, in dem sowohl textexterne Informationen (Metadaten wie Ort, Datum, Land, Legislaturperiode etc.) als auch textinterne Informationen (Auszeichnung von sprachlichen Informationen) kodiert werden.
} 
Bundestagsfraktion, Wahlkreis, Variable Regierung/Opposition, Mandat (direkt gewählt/gewählt über Landesliste). Da die Subkorpora im Deutschen Bundestag, in der französischen Assemblée nationale und im britischen House of Commons unterschiedlich groß sind, ${ }^{7}$ werden die Ergebnisse standardisiert.

\section{Häufigkeit und Verteilung der Pronomina man, on und one im Korpus: Einblick in quantitative Ergebnisse}

Die Korpussuche wurde mit der Software $\mathrm{TXM}^{8}$ anhand der Lemmata durchgeführt. Aus Tabelle 1 gehen die Häufigkeit und Verteilung der Pronomina man, on, one und generisches you im Vergleich zu Referenzkorpora in den untersuchten Sprachen hervor.

Tab. 1: Verteilung von man, on, one und generisches you in Parlamentsdebatten im Vergleich zu Referenzkorpora

\begin{tabular}{lllllll}
\hline & UK & BNC & DE & DWDS & FR & FRANTEXT \\
\hline $\begin{array}{l}\text { Anzahl der Okk. } \\
\text { im Korpus }\end{array}$ & $24+36$ & 20.629 & 1031 & 273.674 & 271 & 1.313 .917 \\
\hline $\begin{array}{l}\text { Anzahl der Tokens } \\
\text { im Korpus }\end{array}$ & 188.913 & 100.000 .000 & 417.095 & 1.550 .082 .127 & 137.620 & 297.385 .241 \\
\hline $\begin{array}{l}\text { Anzahl der Okk./ } \\
\text { 10.000 Tokens }\end{array}$ & 3,17 & 2,06 & 24,72 & 1,77 & & \\
\hline $\begin{array}{l}\text { Häufigkeit der } \\
\text { Okk. im Korpus }\end{array}$ & $0,03 \%$ & $0,03 \%$ & $0,25 \%$ & $0,02 \%$ & $0,2 \%$ & $0,44 \%$ \\
\hline
\end{tabular}

7 Das deutsche Korpus ist das umfangreichste: 225 Sprecher/innen für 417.095 Tokens. Im französischen Korpus gibt es 159 Sprecher/innen für 137.620 Tokens. Im britischen House of Commons hingegen finden sich 302 Sprecher/innen, obwohl das Korpus deutlich kleiner ist (188.913 Tokens).

8 http://textometrie.ens-lyon.fr (Stand: 29.4.2017). Siehe Heiden (2010). 
Große Unterschiede sind erkennbar, denn in den deutschen und französischen Subkorpora sind alle Okkurrenzen ${ }^{9}$ für die Analyse relevant, wohingegen im englischen Fall nur 24 Okkurrenzen von one (325 insgesamt) als Personalpronomina auftraten, weshalb lediglich 7,38\% dieser Okkurrenzen in diese Studie integriert werden. Diese Ergebnisse stehen im engen Zusammenhang mit dem Verhältnis im British National Corpus (BNC), in dem one als Personalpronomen ca. 7\% der Okkurrenzen umfasst (Mignot 2015: 277). Das entspricht 0,012\% der Gesamtanzahl von Tokens im Korpus, was zweimal weniger ist als im BNC (Mignot 2015: 277) - ein Ergebnis, welches sich auf die Korpusgröße zurückzuführen lässt. Wenn das vorliegende britische Korpus mit dem umfangreichen Hansard Korpus $^{10}$ verglichen wird, welches ausschließlich aus Parlamentsdebatten aus Großbritannien besteht, fällt auf, dass one als Personalpronomen beinahe so häufig im politischen Diskurs vorkommt wie in einem nicht-spezialisierten Korpus.

Darüber hinaus wurden 36 Okkurrenzen vom generischen you manuell aussortiert (67 Okkurrenzen insgesamt): Das Pronomen you mit generischer Interpretation stellt 0,2\% der Tokens im Gesamtkorpus und 57,73\% der Okkurrenzen von you dar. Der hohe Anteil generischer Verwendungen ist eine Besonderheit der britischen House of Commons, wo deiktisches you nahezu nicht vorhanden ist (siehe Ilie 2005: 179 in Abschn. 1). In der Tabelle werden die Okkurrenzen von one und generisches you in die gleiche Spalte gestellt.

Wie Tabelle $1 \mathrm{zu}$ entnehmen ist, wird das deutsche Pronomen man wesentlich öfter (annähernd 14 Male mehr) im politischen Korpus (24,72 Okkurrenzen von man pro 10.000 Tokens) als im Referenzkorpus (1,77 Okkurrenzen von man pro 10.000 Tokens) benutzt, was die besondere Rolle von man in zeitgenössischen Parlamentsdebatten hervorhebt. Im Kontrast dazu kommen one und generisch you selten vor. Sie repräsentieren lediglich 0,03\% der Tokens im britischen Korpus, während man und on bei jeweils 0,2\% und 0,25\% liegen und damit achtmal häufiger vorkommen. Der quantitativ signifikante Gebrauch von on im politischen Diskurs wurde für Nicolas Sarkozy herausgearbeitet (Mayaffre 2013) und mit dem populistischen Ansatz des ehemaligen französischen Präsidenten in Verbindung gebracht. Der Vergleich mit FRANTEXT zeigt, dass das Pronomen on nur halb so häufig im politischen Korpus als in literarischen Werken (die den Kern des FRANTEXT-Korpus bilden) verwendet wird. Dieser Befund würde Mayaffres Behauptung bestätigen, wonach on zu informell für politische

$9 \mathrm{Im}$ deutschen Korpus entsprach nur eine Okkurrenz von man einem anderen Wort, nämlich dem englischen Substantiv man in einem auf Englisch ausgesprochenen Satz.

10 Das Hansard Korpus umfasst alle britischen Parlamentsprotokolle zwischen 1803 und 2005 (www.hansard-corpus.org, Stand: 29.4.2017). 
Reden sei. Weil aber gerade das informelle on, das einem wir entspricht, im Korpus nicht vorkommt (siehe Abschn. 1), ist es schwierig, abschließende Schlussfolgerungen darüber zu ziehen, inwieweit das Pronomen on in Parlamentsdebatten tatsächlich vom „normalen“ Gebrauch abweicht.

Ungeachtet dieser aufschlussreichen Ergebnisse, insbesondere was den signifikant hohen Gebrauch von man in Parlamentsdebatten angeht, zeichnen diese Zahlen nur ein grobes Bild. Um die Funktionen der Pronomina man, on, one und generisches you im parlamentarischen Diskurs genauer zu verstehen, wird nun eine Typologie skizziert, die dem Referenzbezug gerecht wird.

\section{Referenzielle Funktionen der Pronomina man, on, one und generisches you im politischen Diskurs}

Obgleich verschiedene Klassifizierungssysteme für die Pronomina man, on und one aus einer einsprachigen Perspektive entwickelt wurden (Wales 1980; Viollet 1988; Zifonun 2000), wurde für den politischen Diskurs hingegen nur eine Typologie vorgeschlagen (Gelabert-Desnoyer 2008). Ursprünglich wurde diese für das spanische Pronomen uno entwickelt, dennoch wird sie für die sprachvergleichende Analyse von Parlamentsdebatten in Deutschland, Frankreich und Großbritannien angewandt, da sie der Besonderheit der Pronomina im politischen Diskurs zurechtkommt. Im Folgenden wird Gelabert-Desnoyers Klassifizierung im Vergleich mit generellen Einblicken über man, on, one und generisches you dargestellt. Dabei wird unterstrichen, wie wichtig es ist, eine einzigartige Typologie für die Referenten im politischen Diskurs zu erarbeiten. Gelabert-Desnoyer unterscheidet zwischen vier referenziellen Funktionen des Pronomens uno:

1. Wenn die Pronomina mit allgemeinem Personenbezug (omnipersonal) $[ \pm$ Sprecher/in] verwendet werden, drücken sie aus, dass der Referent irgendjemand sein kann. Diese Kategorie wurde aus einer monolingualen Perspektive bereits umfangreich aufgelistet (Viollet 1988: 72; Wales 1980: 85; Zifonun 2000: 241); sie entspricht einem generischen Gebrauch.

2. Der selbstreferenzielle Bezug (self-referential) [+ Sprecher/in, - andere Referenten] gibt an, dass der/die Sprecher/in der einzige Referent ist. ${ }^{11}$

11 Für Wales (1980: 85) entspricht diese Kategorie der Bezeichnung 'advanced egocentric' $(I+$ eventuell andere, die mir gleich sind); Zifonun (2000: 241) spricht von einem man ,bezogen auf eine Person, sprecherinklusiv ('ich')“. 
3. Der selbstreferenzielle Erfahrungsbezug (self-referential experiential) [+ Sprecher/in, + andere Referenten] hebt hervor, dass die Verbindung zu dem/der Sprecher/in immer noch besteht, ohne exklusiv zu sein. In dieser Lesart können andere Referenten miteingeschlossen werden. ${ }^{12}$

4. Der Bezug auf andere (other-referential) [- Sprecher/in, + andere Referenten] bezeichnet Pronomina, die nur auf die Adressierten gerichtet sind. Gerade in dieser letzten Kategorie, die am häufigsten vorkommt, erweist sich der erwähnte Unterschied zwischen Adressaten und Adressierten als sehr bedeutsam: Wenngleich die traditionellen linguistischen Markierer der Anrede nicht auftauchen, kann aber auf die Adressierten geschlossen werden. In Wales Typologie (1980: 85) ist dieser Aspekt von one, nämlich der Referenzbezug zu anderen, außer Acht gelassen worden. Jedoch spielt diese Referenz im politischen Diskurs eine wichtige Rolle, was eine Typologie spezifisch für den politischen Diskurs bedeutsam macht.

Tabelle 2 stellt die referenziellen Funktionen von man, on, one und generisches you in Parlamentsdebatten dar. Die Werte der letzten Spalte stammen aus Gelabert-Desnoyer (2008: 413). Für jede Sprache wird in der ersten Spalte die Häufigkeit der Okkurrenzen im Korpus dargestellt. In der zweiten Spalte werden die Prozentanteile, d.h. die Verteilung der Okkurrenzen von man, one, generisches you und on je nach referenzieller Funktion angezeigt.

Tab. 2: Referenzielle Funktionen von man, on, one und generisches you in Parlamentsdebatten

\begin{tabular}{lrrrrrrrrrr}
\hline & $\begin{array}{l}\text { Okk. } \\
\text { man }\end{array}$ & $\%$ & $\begin{array}{l}\text { Okk. } \\
\text { one }\end{array}$ & $\%$ & $\begin{array}{l}\text { Okk. } \\
\text { you }\end{array}$ & $\begin{array}{l}\text { Okk. } \\
\text { on }\end{array}$ & $\begin{array}{l}\text { Okk. } \\
\text { uno }\end{array}$ & $\%$ \\
\hline Omnipersonal & 235 & 22,8 & 5 & 20,9 & 9 & 25 & 57 & 21 & 18 & 40,9 \\
\hline Self-referential & 7 & 0,7 & 2 & 8,3 & 0 & 0 & 1 & 0,4 & 1 & 2,3 \\
\hline $\begin{array}{l}\text { Self-referential } \\
\text { experiential }\end{array}$ & 373 & 36,2 & 11 & 45,8 & 20 & 55,5 & 117 & 43,2 & 2 & 4,5 \\
\hline Other-referential & 416 & 40,3 & 6 & 25 & 7 & 19,5 & 96 & 35,4 & 23 & 52,3 \\
\hline Total & 1031 & & 24 & & 36 & & 271 & & 44 & \\
\hline
\end{tabular}

Aus Tabelle 2 geht deutlich hervor, dass die zweite Kategorie (selbstreferenzieller Bezug) kaum vorkommt: In Parlamentsdebatten vermitteln man, on, one und

12 Wales (1980: 85) nennt diese Kategorie 'generic-egocentric' (I+ anyone else/others). 
generisches you vorwiegend ein Bild des/der Anderen. Im Hinblick auf die Beantwortung der Frage nach den Adressierten des politischen Diskurses werden diese wenigen Beispiele im vorliegenden Beitrag nicht ausgewertet, da sie lediglich das Bild des Sprechers oder der Sprecherin hervorrufen. Im Folgenden werden die drei anderen Erscheinungsformen von man, on, one und generischem you analysiert.

\section{Allgemeiner Personenbezug: wenn man, on, one und generisches you auf jede/n zielen können}

Ungefähr ein Viertel der Okkurrenzen von man, on, one und generisches you umfassen eine unbestimmte, ziemlich breite Menge von Referenten. Dieser Gebrauch ist nicht auf den politischen Diskurs beschränkt. Zifonun (2000: 237f.) spricht in diesen Fällen von der „Generizität bzw. Allgemeingültigkeit des Satzes“: man-Ausdrücke „,repräsentieren somit Standardannahmen über bestimmte Objektbereiche“. Eine solche generische Verwendung hängt häufig mit Sprichwörtern oder verallgemeinernden Aussagen zusammen. Charakteristisch für die Analyse von Parlamentsdebatten über Europa ist der Befund, dass die Pronomina in Stellungnahmen über vergangene Ereignisse oder Entscheidungen auftauchen, in denen der Einsatz der Sprecherin oder des Sprechers abgeschwächt wird:

(1) Dr. Angelica Schwall-Düren (SPD) [Mehrheit]: In den Monaten nach dem Jahr 2000, als die Lissabon-Strategie entwickelt worden ist, ist man von einem sehr optimistischen Klima ausgegangen, und zwar im Zusammenhang mit den Entwicklungen im Bereich der New Economy. Jetzt ist es Zeit, eine Zwischenbilanz zu ziehen. (DE 2004.11.11)

(2) Valéry Giscard d'Estaing (UMP) [Mehrheit]: Cette Constitution, dont on parlait timidement il y a un an, [...] est maintenant reconnue comme nécessaire par l'ensemble des membres de la Convention [...]. (FR 2002.12.03)

Diese Verfassung, von der man vor einem Jahr zaghaft gesprochen hat, [...] ist nun als notwendig von allen Mitgliedern des Konvents anerkannt [...]. ${ }^{13}$

Die Zusammensetzung von man und on mit Passivstrukturen (entwickelt worden ist, est maintenant reconnue) mündet in einem Satz ohne erkennbare Sprecher-

13 Die Originalbeispiele wurden von der Autorin ins Deutsche übersetzt. 
instanz: Jetzt ist es Zeit, eine Zwischenbilanz zu ziehen. In beiden Beispielen wird die Distanz vom Sprecher oder von der Sprecherin durch zeitliche Ausdrücke ([i] $n$ den Monaten nach dem Jahr 2000, il y a un an) herbeigeführt. Diese Okkurrenzen sollten jedoch nicht als „Bezug auf andere“ eingestuft werden, da beide Sprecher/innen, Schwall-Düren und Giscard d'Estaing, als potenzielle Referenten in Frage kommen.

Die Lissabon-Strategie, die im Beispiel (1) erwähnt wird, war ein 2000 in Lissabon verabschiedetes Programm, das teilweise als Grundlage für die Agenda 2010 angesehen wird. Die Agenda 2010 enthält eine Reihe von Reformen des deutschen Sozialsystems und Arbeitsmarktes, die unter einer rot-grünen Bundesregierung - an der Schwall-Düren beteiligt gewesen ist - umgesetzt worden sind. Die Agenda 2010 ist mit besonders unpopulären Maßnahmen verbunden (darunter Hartz IV), die im Sommer 2004 unter anderem zu Montagsdemonstrationen führten.

Wenn Schwall-Düren im November 2004 vor diesem Hintergrund eingesteht, dass „man von einem sehr optimistischen Klima ausgegangen [ist]“, berücksichtigt sie die bittere Ernüchterung der Menschen durch eine allgemeine Referenz. Diese wird aber weder ihrer eigenen Verantwortung noch der ihrer Partei und Bundestagsfraktion gerecht. Zudem beinhaltet das Pronomen man einen vagen Referenzbezug zu europäischen Regierungschefs, die die Reform ebenfalls beeinflusst haben und dadurch Verantwortung zugeschrieben bekommen. Dabei entzieht sich Schwall-Düren in gewissem Maße ihrer Rechenschaft, indem sie sich als Teil eines umfangreicheren Referenten versteht. Die Aussage impliziert außerdem, dass sich damals alle - auch die Bürger/innen - die Lissabon-Strategie so vorgestellt haben. Die Lissabon-Strategie schwebte buchstäblich in der Luft (optimistische[s] Klima). Insgesamt umfasst die inklusive man-Referenz eine so große Anzahl von Referenten, dass die Einräumung einer Fehleinschätzung der politischen Lage nicht mehr ausschließlich auf die Sprecherin zurückzuführen wäre.

\section{Selbstreferenzieller Erfahrungsbezug: von der persönlichen Erfahrung zur Verallgemeinerung}

Das Pronomen you (in seiner generischen Lesart) ist besonders häufig mit einem selbstreferenziellen Erfahrungsbezug assoziiert (55,5\%), durch welchen der/die Sprecher/in einen persönlichen Geisteszustand ausdrücken kann, dabei aber gleichzeitig der Aussage einen allgemein gültigen Wert verleiht: 
(3) Mr. David Cameron (Conservative) [Mehrheit]: I certainly agree that the Durban outcome is worth while and it is a staging post towards another global deal, which is very worth while. I am afraid I do not see any contradiction between being incredibly positive and constructive and having a bottom line. When you have a bottom line, it is quite important that you stick to it. (UK 2011.12.11)

(4) Mr. David Cameron (Conservative) [Mehrheit]: Let me say to the right hon. Lady that I went to Brussels wanting a result at 27, but there were safeguards that I believed that Britain needed. Frankly, you can have all the experience of negotiating in the world, but if you are not prepared to say no from time to time, you do not have any influence or power. (UK 2011.12.11)

Da das Ich in (3) und (4) einbezogen wird, aber nicht der einzige Referent ist, erscheint es folgerichtig, diese Okkurrenzen als selbstreferenziellen Erfahrungsbezug einzuordnen. Diese Klassifizierung hebt die doppelte referenzielle Funktion des generischen Pronomens you hervor, welches sich gleichermaßen auf den/die Sprecher/in und den/die Hörer/in bezieht. Trotz verallgemeinernder Ausdrücke (Indefinitartikel $a$, Bedingung mit when, ungenaue Adverbien quite oder Quantifikatoren all the experience, unterbestimmte zeitliche Ausdrücke wie from time to time) zeigt das deiktische Pronomen der 1. Person Singular (I), dass diese Aussagen im Einklang mit dem Ethos des Premierministers stehen, der seine Entscheidungen durch einen Aufruf zu Menschenverstand rechtfertigt. Darüber hinaus bleibt generisches you unbestimmt: Der dadurch entstandene Empathie-Effekt (Gast et al. 2015) - die Einladung, sich in die gleiche Lage wie diejenige des Sprechers hineinzuversetzen - vermittelt eine Selbstverteidigung („Sie hätten ja das gleiche wie ich getan“) sowohl den Bürger/innen als auch den politischen Opponenten gegenüber, die Cameron wegen seiner Unfähigkeit in EU-Verhandlungen angegriffen haben (,,Sie auch, hätten Sie eine große Verhandlungserfahrung, hätten auch beschränkten Einfluss gehabt“).

Ähnliche Verhaltensmuster sind auch im deutschen Korpus erkennbar. In (5) ist das Pronomen man in der Erfahrung des Sprechers verankert, geht dann aber über die individuelle Einschätzung des Sprechers hinaus, um seine Ansicht als selbstverständlich darzustellen, sobald man „,sich [die Lage] genau anguckt“:

(5) Manuel Sarrazin (BÜNDNIS 90/DIE GRÜNEN) [Opposition]: Dann gibt es diese bilateralen Verträge, die das neue Lieblingskind von Frau Merkel und scheinbar erlösungsbringend sind. Wenn man sich diese genau anguckt, dann erkennt man: Das ist eine neue Scharade. In Wirklichkeit degradieren Sie die europäischen Mechanismen [...]. (DE 2013.12.18) 
Die Auslegung der man-Referenz ist aus der in freier indirekter Rede (auch als erlebte Rede bekannt) formulierten Aussage Sarrazins „Das ist eine neue Scharade“ abzuleiten. Sarrazin ist ein harter Angreifer von Merkels europäischer Politik. Indem er nicht eindeutig behauptet, es sei eine Scharade, fordert der Sprecher die Bürger/innen auf, europäische Abkommen noch gewissenhafter zu beachten, nämlich so, wie er dies tue. Weiterhin suggeriert Sarrazin, dass jede/r zur gleichen Schlussfolgerung kommen würde, womit seine partikuläre Position $\mathrm{zu}$ einer allgemein zutreffenden Behauptung erweitert werden kann. Indirekter Adressierter ist zudem die politische Opposition, die im nächsten Satz durch das gesichtsbedrohende Pronomen Sie (face threatening act) zur erneuten Berücksichtigung des Vertrags angehalten wird.

\section{Der Bezug auf andere Personen: wenn man, on, one und generisches you persönlich werden}

Abschließend unterstreicht der Bezug auf andere, inwiefern die vier Pronomina nicht unpersönlich sind: Obwohl die Semantik der Pronomina keinen bestimmten Referenten adressiert, ist der Ko-Text in dieser Hinsicht eindeutig:

(6) Renate Künast (BÜNDNIS 90/DIE GRÜNEN) [Opposition]: Sie haben sich selber entlarvt - nicht dass wir es nicht schon vorher gewusst hätten -, indem Sie, nachdem Sie so breit die Probleme eines Beitritts der Türkei erörtert haben, bei Kroatien als guter Katholik gleich Ja gesagt haben. [...] Ich will gar nicht negieren, dass Kroatien weit entwickelt ist. Aber, Herr Ramsauer, so kann man Europa, die Erweiterung der Europäischen Union und eine europäische Nachbarschaftspolitik nicht entwickeln; damit kommen Sie den europäischen Interessen nicht nach. (DE 2006.12.14)

(7) Jean-Louis Bianco (Socialiste) [Opposition]: Comment faire partager aux autres une vision de l'Europe quand on n'en a pas soi-même une ! (FR 2005.12.13)

Wie kann man den anderen eine Vision Europas vermitteln, wenn man selber keine hat!

In beiden Beispielen, aber besonders in (6) ist es schwierig zu behaupten, Renate Künast habe durch die man-Referenz auf einen konkreten Referenten verzichtet, denn ihr Hauptopponent (Herr Ramsauer) wird explizit genannt. Das Pronomen man geht über die Einzelperson von Ramsauer (CSU) hinaus, indem jede/r kon- 
servative Politiker/in und sogar jede/r Bürger/in, der/die über die gleichen Eigenschaften verfügt (d.h. „ein guter Katholik“ sein), einbezogen wird. Im politischen Diskurs, in dem der Kontext meist ausreicht, um die durch Unbestimmtheit gekennzeichnete Semantik des Pronomens man zu kompensieren, wird die zweideutige Semantik des Pronomens bewusst strategisch genutzt, damit die konkret angegriffene Person ein/e Vertreter/in wird.

Die Pronomina der 3. Person führen auch argumentative Schemata ein, durch welche eine mögliche andere Meinung erstmals eingeräumt wird, um sie in einem zweiten Schritt zu unterminieren:

(8) Mr. David Cameron, Conservative [Mehrheit]: I quite understand why particularly the Germans want this fiscal union and want tougher rules because they do not want to see irresponsible behaviour repeat itself. One can debate whether that actually requires change in the treaty or not, but we have to spend more time on the other parts of solving the crisis, which are to do with short-term changes and longer-term competitiveness. (UK 2011.12.12)

(9) Édouard Balladur (UMP) [Mehrheit]: En dépit de la volonté de certains pays, tel le Royaume-Uni, partisan permanent de la dilution de l'Europe, la France, pour le moment, doit dire non à tout nouvel élargissement. Applaudissements sur de nombreux bancs du groupe de l'UMP et du groupe UDF On peut trouver cela injuste pour les pays actuellement candidats, mais c'est une nécessité vitale. (FR 2006.12.12)

Trotz des Willens anderer Länder wie Großbritannien, das andauernd für die Schwächung Europas plädiert, muss Frankreich zu jeder neuen Erweiterung vorerst 'Nein' sagen. Beifall bei der UMP sowie bei der UDF Man darf es gegenüber den derzeitigen Kandidatenländern wohl für unfair halten, aber es ist unerlässlich.

Insofern handelt es sich nur um eine Pseudo-Einräumung, die zeigt, dass Themen wie strengere Regelungen (tougher rules) in (8) oder der EU-Beitritt neuer Länder in (9) für Cameron und Balladur jeweils nicht von großer Bedeutung sind. Im Gegensatz dazu wird die Stellungnahme des man durch eine Notwendigkeit (we have to in (8), c'est une nécessité vitale in (9)) ersetzt. Ohne andere Interessen zu vernachlässigen, rückt die Position des Sprechers in den Vordergrund.

Nach der Korpusanalyse der Abschnitte 5, 6 und 7 wird versucht, rückblickend die Frage des vorliegenden Beitrags zu beantworten: Inwiefern sind man, on, one und generisches you generisch, unpersönlich, indefinit? 


\section{Generisch, unbestimmt, persönlich - Zur Referenzaktivierung der Pronomina man, on, one und generisches you in Parlamentsdebatten}

In der Literatur zur Semantik rücken in Definitionsversuchen der Pronomina man, on, one und generisches you drei Hauptaspekte in den Vordergrund: Diese Pronomina werden meistens als nicht-referenziell (vs. referenziell), unpersönlich (vs. persönlich) und indefinit (vs. definit) beschrieben.

In erster Linie wird die Frage nach der Referenz/Referenzialität (beide Termini werden verwendet) beleuchtet. Laut Siewierska (2011: 57f.) sind man, on und one aus einer sprachübergreifenden Perspektive 'R-impersonals', oder „nicht-referenzielle Ausdrücke, die sich durch einen reduzierten Referenzbezug kennzeichnen lassen“14 , wobei der Buchstabe 'R' für 'Referenz' steht. 'R-impersonals' bilden eine Kategorieheterogener Markierer, diedem Anscheinnach wiePersonalkonstruktionen fungieren, jedoch einen menschlichen, aber nicht referenziellen Bezug haben. Obwohl die Pronomina nicht (immer) auf spezifische Individuen hinweisen, hat der vorliegende Beitrag versucht zu zeigen, inwiefern man, on und one nicht als ‘R-impersonals’ oder als „nicht-referenziell“ bezeichnet werden können.

Die Unterscheidung zwischen referenzieller und nicht-referenzieller Verwendung, die z.B. von Bhat (2004: 215) oder Payne/Huddleston (2002: 427) aufgegriffen wird (siehe Mignot 2015: 285), erscheint als problematisch, da sogenannte „nicht-referenzielle“ Verwendungen eigentlich referieren, wie alle Okkurrenzen deutlich gemacht haben. Obwohl der Referenzbezug ungenau bleibt, verweisen die Pronomina mindestens auf eine Menge unbestimmbarer Leute. Eher als ,nichtreferenziell“" können man, on, one und generisches you im politischen Diskurs als „generisch“ beschrieben werden. Wie im Abschnitt 5 vor Augen geführt wurde, entspricht dieser generischen Lesart die Kategorie „allgemeiner Personenbezug“.

Zweitens kann die Frage nach dem „unpersönlichen“ Referenzbezug der Pronomina neu aufgeworfen werden. Eine Lesart von man, on und one oder generisches you, bei der weder die Sprecher/in- noch die Adressatenrollen einbezogen werden, ist zwar theoretisch möglich. Im Korpus referieren aber die Pronomina hauptsächlich auf konkrete, erkennbare Referenten - wenn nicht auf einen einzigen genauen Referenten, der als stellvertretend für eine Gruppe gilt. Vor diesem Hintergrund stellt der Begriff der „Nicht-Person“ (Benveniste 1966: 254) Probleme

14 Originalzitat: ,impersonals triggered by a reduction in referentiality“. 
dar; deshalb werden anschließend an Payne/Huddleston (2002: 426) und Mignot (2015: 279) die Pronomina der 3. Person in das Paradigma der Personalpronomina inkludiert.

Wie De Cock (2014: 191) erläutert, sei das Label „unpersönlich“ (impersonal) problematisch, insbesondere, weil der Begriff sehr unterschiedlich und breit verwendet wird. Für einige bedeutet das, dass die Pronomina keine konkreten Individuen oder Gruppen von Individuen umfassen, für andere bedeutet es, dass die referenzielle Interpretation irgendjemanden oder jede/n enthält. Als gemeinsamer Nenner gilt die semantische Eigenschaft [- spezifisch], d.h. keine konkreten Individuen oder Gruppen von Individuen. Schwer $\mathrm{zu}$ verstehen ist, warum die Pronomina man, on und one und generisches you aus diesem Grund als ,unpersönlich“ beschrieben werden sollten: Sie referieren doch immer auf eine Person (oder Gruppe) und verweisen in dieser Hinsicht auf die semantische Eigenschaft [+ Person], obwohl diese Person nicht unbedingt Teil der unmittelbaren Kommunikationssituation ist oder nicht-spezifisch bleibt. In Parlamentsdebatten ist die Diskrepanz zwischen Adressaten und Adressierten in dieser Hinsicht aufschlussreich (siehe Abschn. 2). Obwohl die Pronomina die Referenten des politischen Diskurses nicht direkt ansprechen oder benennen, referieren sie auf eben jene Personen, die zu den Adressierten des politischen Diskurses gehören.

Schließlich wird folgende Frage aufgeworfen: Sind man, on, one und generisches you indefinite (oder unbestimmte) Pronomina? Wie Mignot (2015: 286) für das Pronomen one betont, ist Generizität nicht automatisch mit Unbestimmtheit verbunden. Die Pronomina man, on und one kombinieren aber beide Eigenschaften: Sie sind generisch und unbestimmt. Hier kann im Nachhinein die Definition von Payne/Huddleston (2002: 371) herangezogen werden: Mit indefiniten Ausdrücken bleibt die Aktivierung der Referenz unklar, während definite Markierer dem/der Hörer/in erlauben, einen präzisen Referenten auszuwählen.

Aus diesem Überblick lässt sich ablesen, dass die Pronomina man, on, one und you Personalpronomina sind, die generisch verwendet werden können und damit eine unbestimmte, potenziell unbegrenzte Lesart vorweisen. Abschließend kann Borthens Aussage (2010: 1812) über Pluralpronomina und die kognitive Zugänglichkeit bzw. die Aktivierung des Referenten für die Hörer/innen aufgegriffen werden: „Warum würde sich jemand wünschen, dass die Referenz vage bleibt?“..15 Anhand eines kontrastiven Korpus von Parlamentsdebatten über Europa in Deutschland, Frankreich und Großbritannien wurde gezeigt, inwiefern das Erwähnen von ratifizierten Teilnehmer/innen ein Zugehörigkeitsgefühl schaffen und politische Opponenten angreifen kann. Obwohl die Pronomina

15 Originalzitat: „Why would anyone intend reference to be vague?“. 
man, on und one syntaktisch zum Paradigma der 3. Person gehören, ist der Referenzbezug keinesfalls auf eine „Nicht-Person“ beschränkt. Vielmehr können die Pronomina auf eine 1. Person (ich, wir) verweisen, die entweder allgemein (allgemeiner Personenbezug) bleibt oder eine Erweiterung der Sprecherin oder des Sprechers darstellt (selbstreferenzieller Erfahrungsbezug). Eine andere wichtige, oft unbeachtete Funktion des Pronomens liegt in seiner Fähigkeit, auf eine spezifische Person(engruppe) hinzuweisen (Bezug auf andere). In diesem Zusammenhang ruft das Pronomen ein Bild der Adressierten politischer Reden hervor, ohne jedoch jene Personen unmittelbar anzusprechen.

Die Beschränkung auf ein Korpus von Parlamentsdebatten veranschaulicht, dass die referenziellen Funktionen der Pronomina man, on, one und generisches you weitgehend vergleichbar sind. Aus der kontrastiven Diskursanalyse geht hervor, dass die oft verwendeten Termini „unpersönlich“ oder „nicht-referenziell“ im politischen Diskurs ungeeignet sind, da die Pronomina man, on, one und generisches you eine erhebliche Rolle bei der Identifizierung von anderen Referenten spielen.

\section{Literatur}

Altenberg, Bengt (2005): The generic person in English and Swedish: A contrastive study of one and man. In: Languages in Contrast 1. 93-120.

Bayley, Paul (Hg.) (2004): Cross-Cultural Perspectives on Parliamentary Discourse. (= Discourse Approaches to Politics, Society and Culture). Amsterdam/Philadelphia: Benjamins.

Benveniste, Émile (1966): Problèmes de linguistique générale I. Paris: Gallimard.

Bertrand, Yves (2002): On, man et les autres. In: Nouveaux cahiers d'allemand 20. 189-209.

Bhat, Darbhe N.S. (2004): Pronouns. Oxford: Oxford University Press.

Borthen, Kaja (2010): On how we interpret plural pronouns. In: Journal of Pragmatics 7. 1799-1815.

Burkhardt, Armin/Pape, Kornelia (Hgg.) (2000): Sprache des deutschen Parlamentarismus: Studien zu 150 Jahren parlamentarischer Kommunikation. Wiesbaden: Springer.

De Cock, Barbara (2014): Profiling Discourse Participants: Forms and functions in Spanish conversation and debates. (= Pragmatics \& Beyond New Series 246). Amsterdam/ Philadelphia: Benjamins.

Ducrot, Oswald (1980): Analyse de textes et linguistique de l'énonciation. In: Ducrot et al.: Les mots du discours. Paris: Editions de Minuit. 7-56.

Fløttum, Kjersti/Jonasson, Kerstin/Norén, Coco (2007): ON : pronom à facettes. Brüssel: De Boeck/Duculot.

Gast, Volker et al. (2015): Impersonal uses of the second person singular: A pragmatic analysis of generalization and empathy effects. In: Journal of Pragmatics 88. 148-162.

Gelabert-Desnoyer, Jaime (2008): Not so impersonal: Intentionality in the use of pronoun uno in contemporary Spanish political discourse. In: Pragmatics 3. 407-424.

Haspelmath, Martin (2001): Indefinite Pronouns. Oxford: Clarendon Press. 
Heiden, Serge (2010): The TXM Platform: Building Open-Source Textual Analysis Software Compatible with the TEI Encoding Scheme. Institute for Digital Enhancement of Cognitive Development. Waseda University. (https://halshs.archives-ouvertes.fr/halshs-00549764/ document, Stand: 23.10.2017). 389-398.

Ilie, Cornelia (2005): Politeness in Sweden: Parliamentary Forms of Address. In: Hickey, Leo/ Stewart, Miranda (Hgg.): Politeness in Europe. (= Multilingual Matters 127). Clevedon: Multilingual Matters. 174-188.

Ilie, Cornelia (Hg.) (2010): European Parliaments Under Scrutiny: Discourse Strategies and Interaction Practices. Amsterdam/Philadelphia: Benjamins.

Johansson, Stig (2007): The generic person in English, German, and Norwegian. In: Johansson, Stig (Hg.): Seeing Through Multilingual Corpora: On the Use of Corpora in Contrastive Studies. (= Studies in Corpus Linguistics 26). Amsterdam/Philadelphia: Benjamins. 175-196.

Kitagawa, Chisato/Lehrer, Adrienne (1990): Impersonal uses of personal pronouns. In: Journal of Pragmatics 5. 739-759.

Kluge, Bettina (2016): Generic uses of the second person singular - how speakers deal with referential ambiguity and misunderstandings. In: Pragmatics 3. 501-522.

Koester, Almut (2010): Building small specialised corpora. In: O’Keeffe, Anne/McCarthy, Michael (Hgg.): The Routledge Handbook of Corpus Linguistics. London/New York: Routledge. 66-79.

Malamud, Sophia A. (2012): Impersonal indexicals: one, you, man, and $d u$. In: The Journal of Comparative Germanic Linguistics 1. 1-48.

Mayaffre, Damon (2013): Sarkozysme et populisme. Approche logométrique du discours de Nicolas Sarkozy (2007-2012). In: Mots. Les langages du politique 103. 73-87.

Mignot, Elise (2015): Pragmatic and stylistic uses of personal pronoun one. In: Gardelle, Laure/ Sorlin, Sandrine (Hgg.): The Pragmatics of Personal Pronouns. Amsterdam/Philadelphia: Benjamins. 275-310.

Payne, John/Rodney, Huddleston (2002): Nouns and noun phrases. In: Huddleston Rodney/ Pullum Geoffrey K. (Hgg.): The Cambridge Grammar of the English Language. Cambridge: Cambridge University Press. 323-523.

Quirk, Randolph et al. (1985): A Comprehensive Grammar of the English Language. London: Longman.

Rissanen, Matti (1967): The Uses of ONE in Old and Early Middle English. Helsinki: Mémoires de la Société Néophilologique.

Siewierska, Anna (2011): Overlap and complementarity in reference impersonals. Man-constructions vs. third person plural-impersonals in the languages of Europe. In: Malchukov, Andrej/Siewierska, Anna (Hgg.): Impersonal Constructions: A Cross-linguistic Perspective. (= Studies in Language Companion Series 124). Amsterdam/Philadelphia: Benjamins. 57-90.

Viollet, Catherine (1988): Mais qui est on? Étude linguistique des valeurs de on dans un corpus oral. In: Linx 1. 67-75.

Wales, Katie (1980): "Personal" and "indefinite" reference: The uses of the pronoun one in present-day English. In: Nottingham Linguistic Circular 9. 93-117.

Zifonun, Gisela (2000): „Man lebt nur einmal.“ Morphosyntax und Semantik des Pronomens man. In: Deutsche Sprache 28. 232-253. 
